\title{
DETERMINACIÓN DEL POTENCIAL BIOQUÍMICO DE METANO (PBM) DE RESIDUOS DE FRUTAS Y VERDURAS EN HOGARES
}

\author{
Claudian SÁNCHEZ-REYES ${ }^{1}$, María Eugenia PATIÑO-IGLESIAS ${ }^{2}$, José Luis ALCÁNTARA-FLORES ${ }^{2 *}$, \\ Yasmi REYES-ORTEGA², María Ana PÉREZ-CRUZ ${ }^{3}$ y Esiquio ORTÍZ-MUÑOZ1 ${ }^{1}$
}

${ }^{1}$ Facultad de Ingeniería Química, Benemérita Universidad Autónoma de Puebla. Bulevar 18 Sur y Avenida San Claudio, Colonia San Manuel, Puebla, Puebla, México, C.P. 72570

${ }^{2}$ Centro de Química-Instituto de Ciencias, Benemérita Universidad Autónoma de Puebla. Bulevar 18 Sur y Avenida San Claudio, Colonia San Manuel, Puebla, Puebla, México, C.P. 72570

${ }^{3}$ Facultad de Ciencias Químicas, Benemérita Universidad Autónoma de Puebla. Bulevar 18 Sur y Avenida San Claudio, Colonia San Manuel, Puebla, Puebla, México, C.P. 72570

*Autor para correspondencia: jlaf7@yahoo.com.mx

(Recibido junio 2015; aceptado octubre 2015)

Palabras clave: digestión anaerobia, biogás, inóculo, sustrato

\section{RESUMEN}

En este trabajo se estudia mediante el proceso de digestión anaerobia (DA), la descomposición de materia orgánica de residuos alimenticios de cocina (sustrato) con lodo (inóculo) de una planta de tratamiento de aguas residuales con la finalidad de obtener biogás y determinar el potencial bioquímico de metano (PBM) al final del proceso. Los ensayos que se llevaron a cabo fueron con relaciones sustrato:inóculo (S:I) 1:2 y 1:3, en términos de sólidos volátiles (SV). En el transcurso de la DA en condiciones mesofílicas $\left(35^{\circ} \mathrm{C}\right)$ y tiempos de residencia (TR) de 15 días, se midieron los parámetros más importantes que influyen en esta digestión: SV, sólidos totales (ST), demanda química de oxígeno (DQO) y $\mathrm{pH}$. El biogás obtenido se purificó por medio de la eliminación de $\mathrm{H}_{2} \mathrm{~S}$ y $\mathrm{NH}_{3}$ con trampas de $\mathrm{NaOH}$ y $\mathrm{H}_{2} \mathrm{SO}_{4} 1 \mathrm{M}$. En el caso de los experimentos con inóculo y sustrato se recuperaron en promedio $1.235 \mathrm{~L}$ de biogás con un $80.15 \%$ de $\mathrm{CH}_{4}$ y en los ensayos con inóculo sin sustrato $0.720 \mathrm{~L}$ de biogás con $90.47 \%$ de $\mathrm{CH}_{4}$. La cuantificación de este gas se llevó a cabo por cromatografía de gases. Los resultados que se obtuvieron concuerdan favorablemente con la producción de biogás y la disminución de la materia o carga orgánica expresada como DQO. En la determinación de PBM se obtuvieron 124.82 y $127.89 \mathrm{~mL} \mathrm{CH}_{4} / \mathrm{g}$ SV para experimentos S:I-1:3 y para la relación $1: 2,103.39$ y $116.27 \mathrm{~mL} \mathrm{CH}_{4} / \mathrm{g} \mathrm{SV}$.

Key words: anaerobic digestion, biogas, inoculum, substrate

\begin{abstract}
In this work we studied the decomposition of organic matter of food kitchen wastes (substrate) by sewage sludge (inoculum) from a wastewater treatment plant, through the process of anaerobic digestion (AD) in order to obtain biogas and to determine the biochemical methane potential (BMP) at the end of this process. The tests were performed with substrate:inoculum ratios $1: 2$ and 1:3, in terms of volatile solids (VS).
\end{abstract}


The most important parameters that influence AD were VS, total solids (TS), chemical oxigen demand (COD) and $\mathrm{pH}$. They were measured in the course of the process in mesophilic conditions $\left(35^{\circ} \mathrm{C}\right)$ and retention times of 15 days. The biogas obtained was purified by removing $\mathrm{H}_{2} \mathrm{~S}$ and $\mathrm{NH}_{3}$ with $\mathrm{NaOH}$ and $\mathrm{H}_{2} \mathrm{SO}_{4}$ traps. For experiments with inoculum and substrate, $1.235 \mathrm{~L}$ of biogas with $80.15 \% \mathrm{CH}_{4}$ were recovered and for tests without substrate, $0.720 \mathrm{~L}$ of biogas with $90.47 \% \mathrm{of} \mathrm{CH}_{4}$ were gathered. The quantification of this gas was performed by gas chromatography. The results agree with biogas production and organic load decrease, expressed as COD. For experiments S:I, the BMP values obtained were 124.82 and $127.89 \mathrm{~mL} \mathrm{CH}_{4} / \mathrm{g}$ VS, for $1: 3$ and 103.39 and $116.27 \mathrm{~mL} \mathrm{CH}_{4} / \mathrm{g}$ VS for 1:2.

\section{INTRODUCCIÓN}

La digestión anaerobia (DA) es un proceso biológico que en ausencia de oxígeno descompone materia orgánica y cuyo producto principal es el biogás. Este biogás es una mezcla de aproximadamente $65 \%$ de $\mathrm{CH}_{4}$ y $35 \%$ de $\mathrm{CO}_{2}$, aunque también puede contener pequeñas proporciones de otros gases como $\mathrm{H}_{2}, \mathrm{~N}_{2}$, $\mathrm{O}_{2}$ y $\mathrm{H}_{2} \mathrm{~S}$ (Deublein y Steinhauser 2008). En cada paso del proceso la producción de gas y tasas de descomposición de los residuos orgánicos son influenciados por diferentes factores, entre estos ácidos grasos volátiles $(\mathrm{AGV}), \mathrm{pH}$, tiempos de residencia(TR), demanda química de oxígeno (DQO), sólidos totales (ST), sólidos volátiles (SV), temperatura, concentración de sustrato, biomasa, tamaño de partícula del sustrato y relación sustrato/inóculo (S:I) (Deublein y Steinhauser 2008).

Los residuos de cocina comprenden los desperdicios en hogares, establecimientos comerciales y servicios públicos entre otras fuentes. Una comparación de diferentes desechos de alimentos y sus características se expresa comúnmente en términos de carbono disponible $(\mathrm{C})$, hidrógeno $(\mathrm{H})$, nitrógeno $(\mathrm{N})$, oxígeno $(\mathrm{O})$ y azufre $(\mathrm{S})$. La composición elemental de carbohidratos (C-40 \%), proteínas (C-46 \%) y lípidos (C-76\%) indica que cierta cantidad de carbono está disponible en los residuos de alimentos, lo que hace que sean degradables (Iacovidou et al. 2012).

El proceso de DA ha tenido una gran aplicación en el control de diferentes residuos. Por ello, se han desarrollado técnicas de gran utilidad a escala de laboratorio para evaluar la conversión de residuos orgánicos a $\mathrm{CH}_{4}$. La prueba del potencial bioquímico de metano (PBM), sirve para evaluar de forma rápida si un residuo puede ser correctamente degradado y así producir $\mathrm{CH}_{4}$. El valor experimental está dado como $\mathrm{mL} \mathrm{CH}_{4} / \mathrm{g} \mathrm{SV}$ adicionados al reactor. La biodegradabilidad de un sustrato está indicada por el rendimiento de $\mathrm{CH}_{4}$, es decir, por el porcentaje de sólidos que se adicionan o destruyen durante la DA. Aunque no se ha definido un método estándar para determinar el PBM, diversos autores han propuesto procedimientos de ensayo para inferirlo al utilizar diferentes relaciones sustrato:inóculo (S:I) o al modificar las condiciones de temperatura, $\mathrm{pH}$, tamaño de partícula del sustrato y/o agitación (Lesteur et al. 2010).

Con base en lo anterior, en el presente trabajo se utilizan las relaciones sustrato:inóculo 1:2 y 1:3 para determinar al final del proceso cuál tendrá un mejor efecto en los resultados de PBM con la mezcla de residuos elegidos.

\section{MATERIALES Y MÉTODOS}

\section{Materiales y su pretratamiento}

El inóculo utilizado fue lodo activado anaerobio obtenido de un biodigestor mesofílico de la planta de tratamiento de aguas residuales "Atoyac Sur" de la ciudad de Puebla, México. Antes de caracterizar el inóculo se almacenó en frascos de vidrio de $2 \mathrm{~L}$ a $4{ }^{\circ} \mathrm{C}$ durante un tiempo máximo de $48 \mathrm{~h}$. El sustrato fue una mezcla de desechos de cocina de hogares en la ciudad de Puebla y se preparó con $25 \mathrm{~g}$ de residuos de naranja sin pulpa, $25 \mathrm{~g}$ de restos de limón sin pulpa, $25 \mathrm{~g}$ de cáscaras de plátano, $15 \mathrm{~g}$ de capas externas de cebolla, $5 \mathrm{~g}$ de tallos de cilantro y $5 \mathrm{~g}$ de cáscaras de papa. A la mezcla preparada se le realizó un pretratamiento que consistió en triturar $100 \mathrm{~g}$ de ésta con $350 \mathrm{~mL}$ de agua destilada en una licuadora Oster de $500 \mathrm{~W}$. El licuado producido llamado "sustrato base" se pasó a través de un tamiz de $2 \mathrm{~mm}$ con la finalidad de aumentar su velocidad de degradación anaerobia. Posteriormente este sustrato base se diluyó con agua destilada hasta igualar la concentración de SV que tenía el inóculo que se iba a utilizar en los experimentos de DA.

\section{Características del inóculo y el sustrato}

La caracterización del inóculo y el sustrato se llevó a cabo al medir el $\mathrm{pH}$ con un potenciómetro 
Thermo Scientific Orion y al determinar ST, SV y DQO por métodos estándar APHA (2005). Estos parámetros son los más frecuentemente monitoreados en procesos de DA. Los valores obtenidos para el inóculo se reúnen en el cuadro I.

En el caso del sustrato, la medición de parámetros se hizo con el sustrato base y para la determinación de DQO se usó la solución diluida del sustrato base que tenía la misma concentración de SV que el inóculo utilizado en cada experimento. Los valores obtenidos para los parámetros mencionados arriba se muestran en el cuadro II.

\section{Diseño de experimentos}

Para los experimentos 1 y 2 se prepararon mezclas de inóculo con agua destilada (reactores testigo: R1b, $\mathrm{R} 2 \mathrm{~b}$ ) y de inóculo con sustrato (reactores: R1s, R2s) con la misma concentración de SV.

El experimento R1s tuvo una relación S:I 1:3 y se preparó con $175 \mathrm{~mL}$ de sustrato y $525 \mathrm{~mL}$ de inóculo. Para el reactor testigo R1b, se mezclaron $175 \mathrm{~mL}$ de agua destilada y $525 \mathrm{~mL}$ de inóculo.

El experimento R2s, relación S:I 1:2, contenía $233 \mathrm{~mL}$ de sustrato y $467 \mathrm{~mL}$ de inóculo. El reactor testigo R2b, se preparó con $233 \mathrm{~mL}$ de agua y $467 \mathrm{~mL}$ de inóculo.

Cabe mencionar que los experimentos R1s y R2s se realizaron por duplicado y los resultados reportados para éstos son el promedio. Además, se usó cascarón de huevo al inicio de cada uno de dichos experimientos con el objetivo de que funcionara como amortiguador. La cantidad adicionada fue de $1 \mathrm{~g}$ de cascarón por cada $0.100 \mathrm{~L}$ de sustrato agregado.

Cada experimento se incubó en reactores por lotes (batch) de $1 \mathrm{~L}$, los cuales se purgaron previamente con gas $\mathrm{N}_{2}$ durante 30 segundos. Finalmente, los reactores se sellaron para asegurar condiciones anaerobias a $35^{\circ} \mathrm{C}$. El volumen de trabajo fue de $700 \mathrm{~mL}$, agitación magnética de 100 rpm y TR de 15 días.

\section{Métodos analíticos}

El monitoreo de parámetros como $\mathrm{pH}, \mathrm{ST}, \mathrm{SV}$ y DQO se llevó a cabo cada dos o tres días durante 15 días (siete mediciones en total) para estudiar las condiciones de degradación del sustrato en función del tiempo. El biogás obtenido se almacenó en inflables de cloruro de polivinilo (PVC) y se hizo pasar a través de trampas que contenían soluciones de $\mathrm{NaOH}$ y $\mathrm{H}_{2} \mathrm{SO}_{4} 1 \mathrm{M}$, con el fin de eliminar el exceso de $\mathrm{CO}_{2}, \mathrm{H}_{2} \mathrm{~S}$ y $\mathrm{NH}_{3}$.

La cuantificación de $\mathrm{CH}_{4}$ en el biogás se llevó a cabo en un cromatógrafo de gases Perkin Elmer Auto System XL, con detector TCD, columna Altech Carbosphere 80/100 y gas de arrastre He grado cromatográfico.

Los resultados de cada experimento de DA se analizaron en $\mathrm{mL} \mathrm{CH}_{4} / \mathrm{g} \mathrm{SV}$ para evaluar el PBM.

CUADRO I. CARACTERÍSTICAS DEL INÓCULO UTILIZADO EN ESTE TRABAJO Y REPORTADAS EN LA LITERATURA

\begin{tabular}{lccccc}
\hline & $\begin{array}{c}\text { Experimento 1 } \\
\text { S:I 1:3 }\end{array}$ & $\begin{array}{c}\text { Experimento 2 } \\
\text { S:I 1:2 }\end{array}$ & Nieto et al. (2012) & Habiba et al. (2009) Lee et al. (2009) \\
\hline $\mathrm{ST}(\mathrm{g} / \mathrm{L})$ & 26.12 & 29.67 & - & & - \\
$\mathrm{SV}(\mathrm{g} / \mathrm{L})$ & 10.59 & 12.64 & 12 & 4.49 & 20 \\
$\mathrm{DQO}(\mathrm{g} / \mathrm{L})$ & 18.59 & 17.76 & - & 27 & - \\
$\mathrm{pH}$ & 7.22 & 7.74 & 7.5 & 6.98 & 7.8 \\
\hline
\end{tabular}

$\mathrm{ST}=$ sólidos totales, $\mathrm{SV}=$ sólidos volátiles, $\mathrm{DQO}=$ demanda química de oxígeno

CUADRO II. CARACTERÍSTICAS DEL SUSTRATO

\begin{tabular}{lcccc}
\hline & $\begin{array}{c}\text { Experimento 1 } \\
\text { S:I 1:3 }\end{array}$ & $\begin{array}{c}\text { Experimento 2 } \\
\text { S:I 1:2 }\end{array}$ & Komemoto K. et al. (2009) & Habiba et al. (2009) Callaghan F.J. et al. (2002) \\
\hline ST (g/L) & 35.83 & 33.09 & - & - \\
SV (g/L) & 34.6 & 30.44 & $94^{*}$ & 56.1 \\
DQO (g/L) & 35.8 & 36.1 & $279^{+}$ & 72 \\
pH & 3.76 & 3.68 & 4.9 & 5.04 \\
\hline
\end{tabular}

$\mathrm{ST}=$ sólidos totales, $\mathrm{SV}=$ sólidos volátiles, $\mathrm{DQO}=$ demanda química de oxígeno

* \% p/p, ${ }^{+} \mathrm{g} / \mathrm{kg}$ 


\section{RESULTADOS Y DISCUSIÓN}

\section{Sólidos totales (ST)}

El parámetro de ST (así como SV y DQO) es proporcional a la cantidad de materia orgánica degradable en una muestra (Zhang et al. 2007). Los valores para R1s y R2s fueron en cada medición, mayores que sus respectivos reactores testigo, $\mathrm{R} 1 \mathrm{~b}$ y $\mathrm{R} 2 \mathrm{~b}$ debido a la mayor cantidad de materia orgánica presente en el sustrato proveniente de los residuos de frutas y verduras que lo componen. Es importante señalar la forma de las gráficas de variación de ST para los reactores con sustrato en comparación con su respectivo testigo (Fig.1), lo que indica un comportamiento similar a volúmenes idénticos de inóculo agregado, así como que la materia orgánica se va degradando. Se observa que la concentración disminuyó ligeramente en los días 1-4, lo cual se debió a que el inóculo se estaba adaptando al sustrato y a las condiciones de operación de los reactores. Entre los días 4 y 11 hubo una disminución más pronunciada ya que la materia orgánica se degradó para generar biogás. Finalmente, entre los días 11 y 15, las cantidades de ST se mantuvieron aproximadamente constantes por la aparición de la etapa metanogénica, con excepción del reactor R2b en el cual la concentración se mantuvo con poca variación entre los días 4 a 11 .

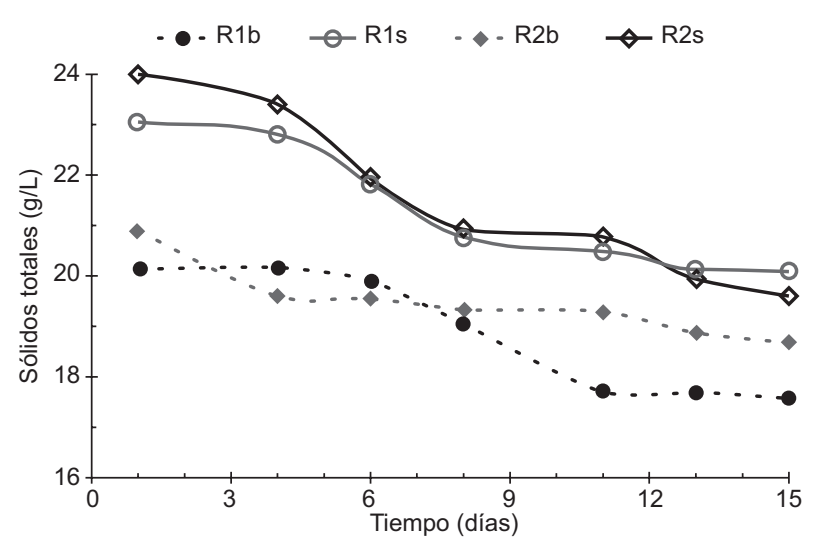

Fig. 1. Comparación de sólidos totales

Experimento 1 (S:I 1:3, R1b-Reactor 1 testigo y R1sReactor 1 con sustrato), Experimento 2 (S:I 1:2, R2bReactor 2 testigo y R2s-Reactor 2 con sustrato)

\section{Sólidos volátiles (SV)}

El comportamiento que presentan las muestras bajo estudio se puede ver en la figura 2. Para todos los reactores la disminución de SV es más notoria entre los días 1 y 4, debido a la degradación anaerobia de la materia orgánica en las etapas hidrolítica,

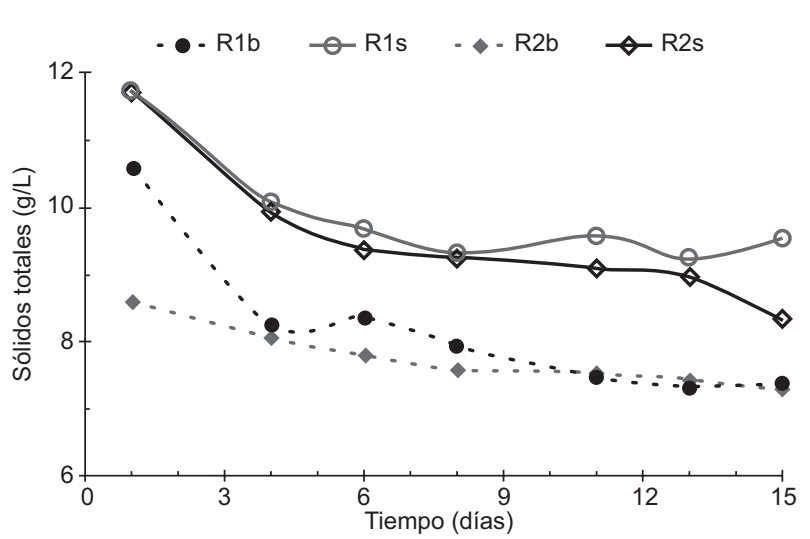

Fig. 2. Comparación de sólidos volátiles (SV) Experimento 1 (S:I 1:3, R1b-Reactor 1 testigo y R1sReactor 1 con sustrato), Experimento 2 (S:I 1:2, R2bReactor 2 testigo y R2s-Reactor 2 con sustrato)

acidogénica y acetogénica, así como a la solubilización de la misma para la producción de biogás (Appels et al. 2008). En el reactor R2b se observó una disminución menos pronunciada debido a la concentración más baja de materia orgánica respecto a los demás reactores. A partir del día 11 los porcentajes de reducción de SV se mantuvieron constantes, debido a la estabilización de la etapa metanogénica y de la tendencia similar en la producción de volumen de biogás (Akunna et al. 2007). En el caso del reactor R1s hubo un ligero aumento en los días 13 a 15 , mientras que en el reactor R2s se presentó una disminución, posiblemente debido a un error de medición relacionado con la observación de que los ST se mantuvieron constantes durante esos días.

La reducción de $\mathrm{SV}$ en los reactores R1b y R1s fue de $30.4 \%$ y $18.5 \%$, respectivamente. Esto sugiere una disminución en la degradación cuando se agregó sustrato al inóculo respecto al inóculo con agua. La disminución de $\mathrm{SV}$ en el caso de R2b y R2s es de $14.9 \%$ y $29.0 \%$, lo que indica un aumento en la degradación cuando se adiciona sustrato al inóculo respecto al testigo.

Forster et al. (2008) reportan una reducción de $32.4 \%$ de SV por DA de residuos de comida durante 20 días; Parkin y Owen (1986) de 40 y 70 \% como reducciones para lodos activados tratados mediante DA por 15 días y ambos dan valores de PBM de 20 a $725 \mathrm{~mL} \mathrm{CH}_{4} / \mathrm{g} \mathrm{SV}$ adicionados en los tiempos mencionados. Los reportes anteriores fijan relaciones S:I similares a las del presente trabajo y aunque obtuvieron valores mayores en la producción de biogás y de PBM, los porcentajes de reducción de SV son comparables a los observados para los reactores R1s $(18.5 \%)$ y R2s $(29.0 \%)$. 


\section{Demanda química de oxígeno (DQO)}

El monitoreo de la DQO en los experimentos es importante porque es un indicador de cuánta materia orgánica se está degradando para ser convertida en biogás (Nayono et al. 2010) y se midió con el propósito de observar el efecto de la relación S:I en la producción final de dicho biogás. La figura 3 muestra la evolución de la DQO en los experimentos realizados, es importante señalar la similitud en la forma de las gráficas entre los reactores R1s y R2s con sus respectivos reactores testigo, $\mathrm{R} 1 \mathrm{~b}$ y $\mathrm{R} 2 \mathrm{~b}$, que indican procesos de adaptación y funcionamiento similares a volúmenes idénticos de inóculo utilizado.

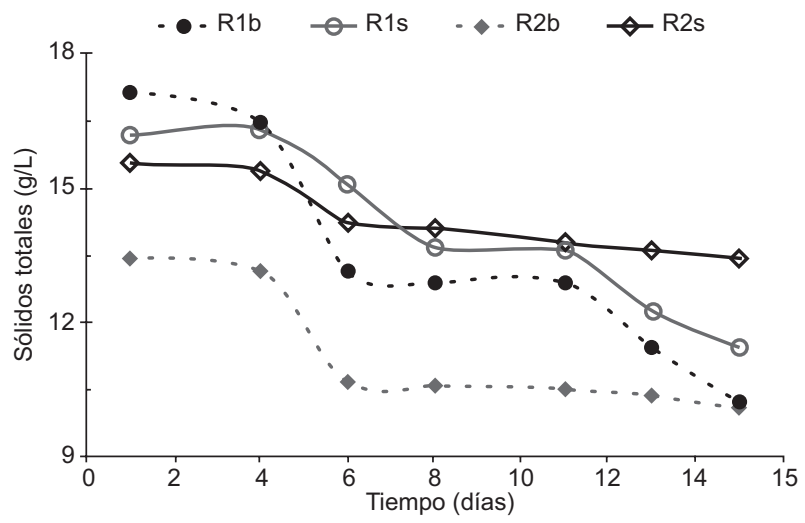

Fig. 3. Comparación de demanda química de oxígeno (DQO) Experimento 1 (S:I 1:3, R1b-Reactor 1 testigo y R1sReactor 1 con sustrato), Experimento 2 (S:I 1:2, R2bReactor 2 testigo y R2s-Reactor 2 con sustrato)

El comportamiento de la DQO en los reactores muestra una disminución significativa en los primeros seis días debido a la hidrólisis y a la solubilización progresiva de materia orgánica por efecto de las enzimas producidas por microorganismos hidrolíticos. En los días 6 a 11 se observó una disminución poco significativa de la DQO por inicio de la etapa metanogénica y en los días 11 a 15 una disminución mayor por estabilización de la etapa metanogénica con una correspondiente transformación de materia orgánica en volumen de biogás. En el período de 11 a 15 días fue más notoria la disminución para R1b y $\mathrm{R} 1 \mathrm{~s}$ debido a la mejor adaptación y funcionamiento del mayor volumen de inóculo agregado en reactores con relación S:I 1:3 en comparación con reactores con relación S:I 1:2. El comportamiento descrito arriba es similar al de los SV (Fig. 2) porque ambos parámetros son proporcionales a la materia orgánica que puede ser convertida a biogás.

Durante los 15 días de DA, la reducción de la DQO (Cuadro III) fue menor en los experimentos R1s y $\mathrm{R} 2 \mathrm{~s}$ en comparación con R1b y R2b. Lo anterior sugiere que el inóculo necesita mayor tiempo de adaptación al sustrato agregado para generar mayor degradación de materia orgánica. Este comportamiento fue similar al observado en la reducción de SV discutido anteriormente.

CUADRO III. REDUCCIÓN DE LA DEMANDA QUÍMICA DE OXÍGENO (DQO) PARA LOS EXPERIMENTOS 1 Y 2

\begin{tabular}{lcccccc}
\hline & & \multicolumn{2}{c}{$\begin{array}{c}\text { Experimento } 1 \\
(\text { S:I 1:3) }\end{array}$} & & \multicolumn{2}{c}{$\begin{array}{c}\text { Experimento 2 } \\
(\text { S:I 1:2) }\end{array}$} \\
\cline { 3 - 4 } \cline { 6 - 7 } & & R1b & R1s & & R2b & R2s \\
\hline $\begin{array}{l}\text { Reducción } \\
\text { de }\end{array}$ & $\mathrm{g} / \mathrm{L}$ & 6.96 & 4.74 & & 3.31 & 1.96 \\
DQO & $\%$ & 40.58 & 29.31 & & 24.64 & 12.62 \\
\hline
\end{tabular}

S:I Sustrato:Inóculo, R1b-Reactor 1 testigo, R1s-Reactor 1 con sustrato, R2b-Reactor 2 testigo, R2s-Reactor 2 con sustrato

\section{pH}

El pH es un parámetro importante en los ensayos de PBM, ya que condiciona el buen funcionamiento de las diferentes poblaciones microbianas, por lo que se midió en todos los reactores usados durante este trabajo. Para evitar la acidificación de los reactores $\mathrm{R} 1$ s y R2s se realizó un control de $\mathrm{pH}$ con la adición de cascarón de huevo por contener $\mathrm{CaCO}_{3}$. El efecto amortigüador del cascarón es prometedor en los reactores, ya que se utilizó un residuo común en las cocinas que mantuvo el $\mathrm{pH}$ cercano a la neutralidad. Esto favoreció a que las bacterias metanogénicas (sensibles a cambios de $\mathrm{pH}$ ) realizaran su función y se produjera $\mathrm{CH}_{4}$ en los reactores.

Los valores de $\mathrm{pH}$ (Cuadro IV) para los reactores R1s y R2s fueron menores que los de sus respectivos testigos, R1b y R2b, debido a las características ácidas del sustrato adicionado. Se observó en los

CUADRO IV. VALORES DE pH PARA LOS EXPERIMENTOS 1 Y 2

\begin{tabular}{cccccc}
\hline & \multicolumn{2}{c}{ Experimento 1 } & & \multicolumn{2}{c}{ Experimento 2 } \\
\cline { 2 - 3 } \cline { 5 - 6 } Días & R1b & R1s & & R2b & R2s \\
\hline 1 & 7.22 & 7.22 & & 8.14 & 7.54 \\
4 & 7.66 & 6.94 & & 8.19 & 7.49 \\
6 & 7.94 & 7.52 & & 7.04 & 7.81 \\
8 & 7.56 & 7.19 & & 8.20 & 7.95 \\
11 & 8.04 & 7.60 & & 8.28 & 7.52 \\
13 & 8.13 & 7.73 & & 8.25 & 7.74 \\
15 & 7.84 & 7.62 & & 8.06 & 7.71 \\
\hline
\end{tabular}

R1b-Reactor 1 testigo, R1s-Reactor 1 con sustrato, R2b-Reactor 2 testigo, R2s-Reactor 2 con sustrato 
primeros una disminución durante los cuatro días iniciales. Dicha disminución es atribuida a la hidrólisis del residuo y a la liberación de ácidos durante la etapa acidogénica (Appels et al. 2008). A partir del día 6 el $\mathrm{pH}$ aumentó prácticamente en todos los reactores, con una excepción, posiblemente por las etapas acetogénica y metanogénica, las que están relacionadas con la estabilización de SV y DQO (Figs. 2 y 3), así como al incremento en la producción de $\mathrm{CH}_{4}$.

El comportamiento del $\mathrm{pH}$ en este trabajo es parecido al que reporta Raposo et al. (2006), donde el intervalo de $\mathrm{pH}$ es de 7.0 a 8.2 para 15 días de prueba, lapso igual al TR de los experimentos de la presente investigación.

\section{Volumen de biogás}

La producción de biogás en los primeros cuatro días fue baja (Fig. 4) debido a la adaptación en los reactores y después se incrementó significativamente con una tendencia similar a partir del día 11. Los reactores R1s y R2s tuvieron mayor producción de biogás debido a la mayor cantidad de materia orgánica contenida en comparación con los reactores testigo en los cuales se agregó agua en lugar de sustrato, comportamiento semejante al reportado por Velmurugan y Ramanujan (2012). Esta evolución coincidió con la mayor disolución de la materia orgánica (DQO y SV) y el consiguiente aumento del $\mathrm{pH}$ debido a la realización de la etapa metanogénica.

El reactor R1s con la proporción S:I 1:3 produjo mayor acumulación de biogás que R2s con un volumen final de 1.260 L. Los volúmenes obtenidos en el presente trabajo son comparables a los que reporta Liu et al. (2009) de 0.358 y $0.784 \mathrm{~L}$ en reactores de 1 L con relación S:I 1:3 y también a los obtenidos por

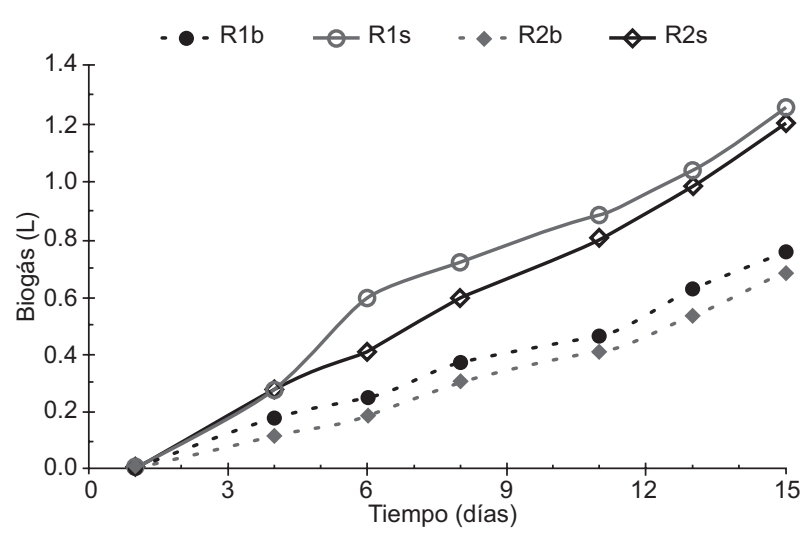

Fig. 4. Acumulación de biogás en los reactores. Experimento 1 (S:I-1:3, R1b-Reactor 1 testigo y R1sReactor 1 con sustrato), Experimento 2 (S:I-1:2, R2bReactor 2 testigo y R2s-Reactor 2 con sustrato)
Velmurugan y Ramanujan (2012) de 0.2 y 0.4 L en reactores de $0.5 \mathrm{~L}$ con relación S:I 1:1 y TR de 19 días.

En resumen, las mezclas S:I-1:3 y 1:2 dieron mayor producción de biogás en una proporción de 67 y 76 $\%$, respectivamente, en comparación con los reactores R1b y R2b los que sólo contenían inóculo más agua.

\section{Cuantificación de $\mathrm{CH}_{4}$ en el biogás por cromato- grafía de gases (CG)}

El porcentaje de $\mathrm{CH}_{4}$ en el biogás se midió al final de los experimentos 1 y 2 por CG, para ello se realizaron mediciones por triplicado para cada muestra recolectada y en todos los casos es biogás previamente lavado. Cabe mencionar que no se consideraron componentes traza como $\mathrm{H}_{2} \mathrm{~S} \mathrm{y} \mathrm{NH}_{3}$ porque se eliminaron en soluciones de $\mathrm{NaOH}$ y $\mathrm{H}_{2} \mathrm{SO}_{4}$. La calidad del gas está directamente relacionada con una mayor cantidad de $\mathrm{CH}_{4}$ que de $\mathrm{CO}_{2}$ (Deublein y Steinhauser 2008), además de que son los productos principales en la reacción bioquímica de DA. En las muestras analizadas se cuantificaron ambos gases y se obtuvo un mayor porcentaje de $\mathrm{CH}_{4}$ en comparación con el $\mathrm{CO}_{2}$ (Cuadro V).

CUADRO V. CANTIDAD DE $\mathrm{CH}_{4} \mathrm{Y} \mathrm{CO}_{2}$ EN EL BIOGÁS DE LOS EXPERIMENTOS 1 Y 2

\begin{tabular}{lcc}
\hline Reactor & $\mathrm{CH}_{4} \%$ & $\mathrm{CO}_{2} \%$ \\
\hline R1b & 91.87 & 8.12 \\
R1s & 75.19 & 24.80 \\
R2b & 89.07 & 10.92 \\
R2s & 85.11 & 14.88 \\
\hline
\end{tabular}

En los reactores testigo, R1b y R2b, se tuvo un mayor porcentaje de $\mathrm{CH}_{4}$ en comparación con sus respectivos reactores con sustrato, R1s y R2s, debido a que las bacterias metanogénicas se adaptan mejor al medio que sólo contiene agua e inóculo. Para los reactores, R1s y $\mathrm{R} 2 \mathrm{~s}$, el mayor porcentaje de $\mathrm{CH}_{4}$ respecto a $\mathrm{CO}_{2}$ pudo deberse a los siguientes factores:

El sustrato se encuentra adecuadamente triturado y homogenizado, acelerando la descomposición, lo cual se reflejó en el monitoreo de SV y DQO donde hubo una disminución durante el proceso de fermentación.

Un mayor contenido de líquido en el reactor disolvió una mayor concentración de $\mathrm{CO}_{2}$ en el agua, reduciendo el nivel de este gas y aumentando la concentración relativa de $\mathrm{CH}_{4}$ en la fase gaseosa.

La cantidad de $\mathrm{CH}_{4}$ para los experimentos 1 y 2 es muy importante para evaluar el PBM y decidir cuál de las dos relaciones $\mathrm{S}: \mathrm{I}$ es más productiva en la generación de $\mathrm{CH}_{4}$. 


\section{Evaluación del potencial bioquímico de metano (PBM)}

Una vez obtenida la cantidad de $\mathrm{CH}_{4}$ en el biogás y la cantidad de SV adicionados en los reactores de los experimentos 1 y 2 se evalúo el PBM con la expresión:

$\mathrm{PBM}=\mathrm{mL} \mathrm{CH}_{4} / \mathrm{g} \mathrm{SV}$ adicionados al reactor

Los resultados de la evaluación de PBM se muestran en el cuadro VI, así como los valores de volumen de biogás, contenido de $\mathrm{CH}_{4}$ (porcentaje y volumen) y SV adicionados en los experimentos estudiados.

De los resultados mostrados en el cuadro VI se ve que el mayor porcentaje de $\mathrm{CH}_{4}$ obtenido es para los reactores testigo. Sin embargo, se recolectó mayor volumen de biogás en los reactores R1s y R2s resultando también mayor volumen de $\mathrm{CH}_{4}$ comparado con los reactores testigo. Encontramos que los valores correspondientes al PBM son mayores para los reactores R1s y R2s debido a la mayor cantidad de materia orgánica disponible (medida como ST, SV o DQO) para su transformación a $\mathrm{CH}_{4}$. El experimento $\mathrm{R} 1 \mathrm{~s}$ es el que tiene mayor valor de PBM explicado por la mejor adaptación y funcionamiento del mayor volumen de inóculo agregado en reactores con relación S:I 1:3, en comparación con la relación 1:2. Sin embargo, podría ser posible que en una prueba de PBM con mayor número de días, el inóculo en el experimento con proporción S:I 1:2 se adapte mejor al mayor volumen de sustrato adicionado y su PBM sea mejorado.

Valores de PBM similares a los obtenidos en la presente investigación son reportados por O-Thong et al. (2012), quienes evalúan el PBM de 40-400 $\mathrm{mL} \mathrm{CH}_{4} / \mathrm{g}$ SV. Por otro lado, Pabón Pereira et al. (2012) evalúan el PBM para residuos de cocina (mostaza y frijoles) y sus resultados son mayores a $140 \mathrm{~mL} \mathrm{CH}_{4} / \mathrm{g} \mathrm{SV}$, con ello se comprueba que en este estudio se obtuvieron resultados prometedores y que los valores de la evaluación de PBM para las dos relaciones S:I fue aceptable.

\section{CONCLUSIONES}

El PBM se determinó a 15 días para dos mezclas con relaciones S:I 1:3 y 1:2, utilizando como sustrato una mezcla de residuos de frutas y verduras obtenidos de cocinas. Como inóculo se empleó un lodo proveniente de una planta de tratamiento de aguas residuales. Los experimentos se monitorearon a través de los parámetros de SV, ST y DQO, los cuales mostraron comportamientos de degradación de materia orgánica similares entre los experimentos con sustrato (R1s y R2s) al compararlos con sus respectivos reactores testigo (R1b y R2b) lo que indica procesos de adaptación y funcionamiento similar a volúmenes idénticos de inóculo adicionado. Con base en el comportamiento de las gráficas de SV, ST, DQO, $\mathrm{pH}$ y volumen de biogás para los experimentos 1 y 2 , se propone que las etapas de hidrólisis, acidogénesis y acetogénesis se efectúan principalmente en el periodo de 1 a 11 días. La estabilización de la etapa de metanogénesis se presenta en los días 11 a 15 , lo que está relacionado con el mayor aumento en la producción de volumen de biogás en este periodo. Una contribución novedosa de este trabajo fue la utilización de un residuo común en cocinas como es el cascarón de huevo para mantener un $\mathrm{pH}$ óptimo durante la DA. Es importante señalar que el mayor valor de PBM a los 15 días fue para el reactor con sustrato R1s (relación S:I 1:3) asignado a la mejor adaptación y funcionamiento del inóculo cuando es agregado un mayor volumen de inóculo y una menor cantidad de sustrato en el reactor, en comparación con el experimento S:I 1:2.

\section{AGRADECIMIENTOS}

Los autores de este trabajo agradecen a la planta de tratamiento de aguas residuales municipales "Atoyac Sur" de la ciudad de Puebla, México, por la aportación de lodo de depuradora, el cual se usó

CUADRO VI. RESULTADOS DEL POTENCIAL BIOQUÍMICO DE METANO (PBM) PARA LOS REACTORES DE LOS EXPERIMENTOS 1 Y 2

\begin{tabular}{lccccc}
\hline Reactor & $\begin{array}{c}\text { Biogás } \\
(\mathrm{L})\end{array}$ & $\begin{array}{c}\mathrm{CH}_{4} \\
\%\end{array}$ & $\begin{array}{c}\mathrm{CH}_{4} \\
(\mathrm{~L})\end{array}$ & $\begin{array}{c}\mathrm{g} \mathrm{SV} \\
\text { adicionados }\end{array}$ & $\begin{array}{c}\mathrm{PBM}\left(\mathrm{mL} \mathrm{CH}_{4} / \mathrm{g} \mathrm{SV}\right. \\
\text { adicionados })\end{array}$ \\
\hline $\mathrm{R} 1 \mathrm{~b}$ & 0.755 & 91.87 & 0.694 & 5.56 & 124.82 \\
$\mathrm{R} 1 \mathrm{~s}$ & 1.260 & 75.19 & 0.947 & 7.41 & 127.80 \\
$\mathrm{R} 2 \mathrm{~b}$ & 0.685 & 89.07 & 0.610 & 5.90 & 103.39 \\
$\mathrm{R} 2 \mathrm{~s}$ & 1.210 & 85.11 & 1.029 & 8.85 & 116.27 \\
\hline
\end{tabular}

$\mathrm{SV}=$ sólidos volátiles 
como inóculo durante la obtención de biogás en el proceso de digestión anaerobia. Asimismo, al Laboratorio de Adsorción y Catálisis de la Facultad de Ciencias Químicas de la Benemérita Universidad Autónoma de Puebla por las determinaciones con cromatografía de gases.

\section{REFERENCIAS}

Akunna J.C., Abdullahi Y.A. y Stewart N.A. (2007). Anaerobic digestion of municipal solid wastes containing variable proportions of waste types. Water Sci. Technol. 56, 143-149. DOI: 10.2166/wst.2007.725.

APHA (2005). Standard methods for the examination of water and wastewater. 21 Ed., Centennial Edition, Washington, EUA, 1368 pp.

Appels L., Baeyens J., Degrève J. y Dewil R. (2008). Principles and potential of the anaerobic digestion of waste-activated sludge. Prog. Energ. Combust. 34, 755-781. DOI: 10.1016/j.pecs.2008.06.002.

Callaghan F.J., Wase D.A.J., Thayanithy K. y Forster C.F. (2002). Continuous co-digestion of cattle slurry with fruit and vegetable wastes and chicken manure. Biomass Bioenerg. 27, 71-77. DOI: 10.1016/S09619534(01)00057-5.

Deublein D. y Steinhauser A. (2008). Biogas from waste and renewable resources. An Introduction. WILEY$\mathrm{VCH}$, Weinheim, Alemania, $443 \mathrm{pp}$.

Forster-Carneiro T., Pérez M. y Romero L.I. (2008). Influence of total solid and inoculum contents on performance of anaerobic reactors treating food waste. Bioresource Technol. 99, 6994-7002. DOI: 10.1016/j. biortech.2008.01.018.

Habiba L., Hassib B. y Moktar H. (2009). Improvement of activated sludge stabilisation and filterability during anaerobic digestion by fruit and vegetable waste addition. Bioresource Technol. 100, 1555-1560. DOI: 10.1016/j.biortech.2008.09.019.

Iacovidou E., Ohandja D.-G. y Voulvoulis N. (2012). Food waste co-digestion with sewage sludge - Realising its potential in the UK. J. Environ. Manage. 112, 267-274. DOI: $10.1016 /$ j.jenvman.2012.07.029.

Komemoto K., Lim Y.G., Nagao N., Onoue Y., Niwa C. y Toda T. (2009). Effect of temperature on VFA's and biogas production in anaerobic solubilization of food waste. Waste Manage. 29, 2950-2955. DOI: 10.1016/j. wasman.2009.07.011.

Lee D.H., Behera S.K., Kim J.W. y Park H-S. (2009). Methane production potential of leachate generated from Korean food waste recycling facilities: A lab-scale study. Waste Manage. 29, 876-882. DOI: 10.1016/j.wasman.2008.06.033.
Lesteur M., Bellon-Maurel V., Gonzalez C., Latrille E., Roger J.M., Junqua G. y Steyer J.P. (2010). Alternative methods for determining anaerobic biodegradability: A review. Process Biochem. 45, 431-440. DOI:10.1016/j. procbio.2009.11.018.

Liu, G., Zhang R., El-Mashad H.M. y Dong R. (2009). Effect of feed to inoculum ratios on biogas yields of food and green wastes. Bioresource Technol. 100, 5103-5108. DOI: 10.1016/j.biortech.2009.03.081.

Nayono S.E., Gallert G. y Winter J. (2010). Co-digestion of press water and food waste in a biowaste digester for improvement of biogas production. Bioresource Technol. 101, 6987-6993. DOI: 10.1016/j. biortech.2010.03.123.

Nieto P.P., Hidalgo D., Irusta R. y Kraut D. (2012). Biochemical methane potential (BMP) of agro-food wastes from the Cider Region (Spain). Water Sci. Technol. 66, 1842-1848. DOI: 10.2166/wst.2012.372.

O-Thong S., Boe K., Angelidaki I. (2012). Thermophilic anaerobic co-digestion of oil palm empty fruit bunches with palm oil mill effluent for efficient biogas production. Appl. Energ. 93, 648-654. DOI: 10.1016/j. apenergy.2011.12.092.

Pabón Pereira C.P., Castañares G. y Van Lier J.B. (2012). An OxiTop $\left({ }^{\circledR}\right)$ protocol for screening plant material for its biochemical methane potential (BMP). Water Sci. Technol. 66, 1416-1423. DOI: 10.2166/wst.2012.305.

Parkin G. F., y Owen W. F. (1986). Fundamentals of anaerobic digestion of wastewater sludges. J. Environ. Eng. 112, 867-920. DOI: 10.1061/(ASCE)07339372(1986)112:5(867).

Raposo F., Banks C. J., Siegert I., Heaven S. y Borja R. (2006). Influence of inoculum to substrate ratio on the biochemical methane potential of maize in batch tests. Process Biochem. 41, 1444-1450. DOI: 10.1016/j. procbio.2006.01.012.

Velmurugan B. y Ramanujam R. (2012). Co-Digestion of tannery solid wastes with primary sewage sludge and vegetable wastes for biogas production. J. Solid Waste Tech. Manag. 38, 11-18. DOI: http://dx.doi. org/10.5276/JSWTM.2012.11.

Zhang R., El-Mashad H.M., Hartman K., Wang F., Liu G., Choate C. y Gamble P. (2007). Characterization of food waste as feedstock for anaerobic digestion. Bioresource Technol. 98, 929-935. 